Nous en revenons ainsi à cr que nous avons dit plus haut. Avant toutes choses, il importe de vérifier les souches auxquelles appartiennent les reproducteurs arc-en-ciel existant dans nos piscicultures. L'élimination s'impose de ceux donnant naissance à des descendants qui, ou bien ne se reproduisent pas dans les cours d'cau où ils furent déversés, ou bien abandonnent ces cours d'cau. Il faut créer une race de Truite arc-en-cicl capable de se développer et de sc reproduire normalement en raux douces. Et, pour cela, on devra s'adresser, à Salmo shasta en certaines situations particulièrement favorables, mais surtout à Salmo lewisi. C'est là un travail de toute première importance que les établisscments de pisciculture de l'Etat devraient être les premiers à entreprendre.

\title{
LES DÉGATS CAUSÉS PAR LA GELÉE DANS LES ÉTANGS DE LORRAINE
}

\author{
Par le Général de MORLAINCOLRT \\ Président de la Fédération des Syndicats des étangs de la région de l'Est
}

Les grands froids du mois de Décembre i 933 ont causé d’assez importants dégâts dans le domaine piscicole lorrain, et nous croyons devoir relater reux qui peuvent comporter des enseignements.

Tout d'abord, il faut remarquer que ces froids sont survenus subitement. ct après un été et un automne où la sécheresse avait été exceptionnelle, de sorte que nombre de ruisscaux alimentant des étangs ne donmaient qu'un apport d'eau insuffisant, ce qui avait fait, soit ajourner an printemps, soit tout au moins retarder, la pêche de certains étangs.

Les propriétaires qui ont ajourné leur pêche au printemps n'ont pas. pour le moment, subi de dommages, mais il est fort possible que leurs étangs ne puissent être qu'incomplètement remplis pour l'ćté $\mathrm{r}_{934}$; d'autre part, la pêche des étangs qui n'avait été que retardéc va se trouver reportée au mois de Mars très probablement, car, à moins d'une température exceptionnellement douce, la grlace de nos ctangs, qui a so centimètres d'épaisseur, ne sera pas fondue de sitôt.

Il y aura donc, à ce moment, afflux de poisson sur le marché lorrain, donc baisse du prix de vente, imputahle en fait aux grands froids.

Mais les dommages les plus sérieux sont ceux qui se sont produits dans les étangs en pêche ou dans les réservoirs.

Il est arrivé notamment qu'un des plus grands étangs de l'Est était en pêche au moment des fortes gelées de Décembre, de sorte que tout le poisson rassemblé dans un espace très limité s'est trouvé pris sous 20 centimètres 
de glace, dans une quantité d'eau insuffisante, ct a péri : la perte est évaluée à 30.000 kilogrammes de poisson.

Nous avons été sur le point d'avoir nous-même un arcident du même ordre, quoique dans une moindre proportion :

Dans un étang de 25 hectares. en piche, et recouvert de 20 centimètres de glace, le garde s'aperçut que le poisson se pressait dans le canal d'alimentation qui recevait encore un peu d'eau; il fit casser la glace sur la largeur de re fossé et, sur une longueur de 60 mètres, put ainsi prendre à la trouble, dans ce fossé. plus d'un millier de Carpettes qui étaient restées dans l'étang, après l'interruption de la pêche par suite des froids.

Nous fûmes moins heureux pour l'un de nos grands réservoirs, malgré la précaution prise d'y casser chaque jour la glace sur une surface de ? mètres carrés environ, pour pouvoir surveiller l'état du poisson, mesurer l'épaisseur de la glace et en déduire le volume d'eau restant dans chaque réservoir, ce qui permet, en principe, de se rendre compte si ce volume cst suffisant pour la quantité de poisson qu'on y a mis avant les froids.

Le garde vovant, par l'owverture ainsi pratiquée dans la glace, le poisson " piper ", agrandit immédiafement celfe ouverture, ef, aver une trouble, enleva roo kilogrammes de Carpe, de sorte qu'il n'y eut aucune perte : mais, ayant eu un empêchement pour venir le lendemain, il trouva, le surlendemain, des poissons morts dans l'ouverture faite dans la glace du réservoir voisin ; la glace avait alors 30 centimètres d'épaisseur et, comme il y eut une perte de temps pour avoir sur place le personnel et les outils nécessaires, la plus grande partie du poisson rui sc trouvait dans ce réservoir fut retirée morte.

Un accident du même genre est arrivé à un propriétaìre d'étangs de la Moselle, mais beaucoup plus important, car il se chiffre par une perte de ro.ooo kilogrammes de Carpes.

Par contre. nous pouvons signaler que semblahle mortalité a ćté évitée dans les grands réservoirs de l'Etang de la Chausséc (Meuse), en découpant avec une scie de boucher, sur lo pourtour de ces réservoirs, une hande de glace de r m. 5o de largeur, ce qui a permis, après avoir cassé, puis enlevé avec des fourches à pierre res blocs de glace. de passer, sous le bloc de glace resté dans le milieu du réservoir. un filet traînant avec lequel on put reprendre la plus grande partie du poisson.

La conclusion de ces accidents est la nécessité de surveiller dans les ćtangs en pêche et dans les réservoirs, pendant les grands froids, l'état du poisson resté sous la glace, et, au besoin, l'aćrer l'eau restant sous la glace, soit en y envoyant de l'air avec une pompe, soit, plus simplement, en $\mathbf{y}$ puisant de l'eau pendant un certain temps avec un seau dont on reverse ensuite l'eau dans le réservoir.

Une autre conclusion. c'est qu'il serait bon que nos collègues fissent état de ces accidents, quand ils auront chacun à défendre leurs intérêts dans la nouvelle évaluation du revenu de leurs étangs. 